\title{
Transformation of High-Power Gyrotron Output Radiation Frequency un- der Conditions of Raman Scattering on Additional Electron Beam
}

\author{
L.A. Yurovskiy, N. S. Ginzburg, A.M. Malkin, A.S. Sergeev, I.V. Zotova \\ Institute of Applied Physic RAS, Nizhny Novgorod, Russia, ginzburd@appl.sci-nnov.ru
}

Development of microwave oscillators and of gyrotrons in particular [1] increases the field of potential applications, for which the radiation spectrum control is essential. For example, in gyrotron applications as a source for plasma heating, set frequencies are chosen based on a compromise between the absorption in central and peripheral areas of plasma. Along with this, the investigations show [2] that plasma heating can be much more efficient if the frequency tuning of the generated signal is possible in the gyrotrons involved. In this paper, we propose using the Stimulated Raman Backscattering (SRB) regime on the additional weakly relativistic electron beam. Currently, this regime is under investigations for compression and amplification of optical frequency pulses scattered on the plasma with neglection of frequency transformation [3-5]. However, in microwave frequency range, if the electron beam is used, Doppler shifts values become of the same order as the pump and the scattered signal frequencies. Moreover, the feature of this regime is energy transfer from higher-frequency (HF) pump wave concurrent with the electrons to the lower-frequency (LF) backward propagating scattered wave. Energy transfer, accompanied by the development of the absolute instability, leads to the system self-excitation in the absence of external resonators. This allows for a smooth frequency tuning by varying energy of the electron beam.

The model, where SRB regime take place, is presented in Fig.1. Here the pump wave concurrent with the electrons is scattered on the magnetized cylindrical electron beam in the waveguide, into the backward signal with a frequency downshift. The strong guiding field can increase the transverse oscillations of the electrons in the pump field. However, the magnetic field should be far from the values corresponding to the cyclotron resonance with the pump wave. Otherwise, excitation of parasitic cyclotron oscillations leads to the violation of combination synchronism conditions.

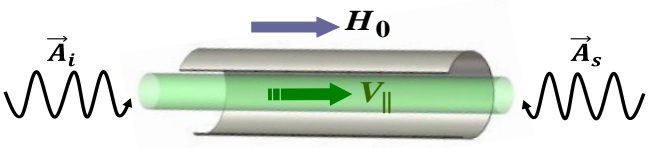

Fig. 1. The model of scattering of the concurrent pump wave into the LF backward wave

In case of sufficiently large density of electron beam, it can be considered as a material medium supporting the space-charge waves. Pump wave leads to the excitation of the Fast Space-Charge Wave (FSCW) possessing positive energy in the absorbing beam. As a result, selecting appropriate combination synchronism detuning, leads to emerging of SRB on space-charge waves with next synchronism conditions:

$$
\omega_{i}-h_{i} V_{\|}-\omega_{s}-h_{s} V_{\|}=\omega_{p}
$$

where $\omega_{p_{\|}}$is the longitudinal plasma frequency, $i, s-$ indexes of pump and scattered waves correspondingly.

This process described by a three-wave interaction equations, which include the equations for a $\mathrm{HF}$ pump wave, an LF scattered wave and a FSCW:

$$
\frac{d A_{b}}{d z}=A_{i} A_{s} \quad \frac{d A_{i}}{d z}=-A_{b} A_{s} \quad \frac{d A_{s}}{d z}=-A_{b} A_{i}
$$

with boundary conditions

$$
\left.A_{b}\right|_{z=0}=\left.0 \quad A_{s}\right|_{z=L}=\left.0 \quad A_{i}\right|_{z=0}=A_{i 0}
$$

where $A_{b}, A_{i}, A_{s}$ - normalized amplitudes of spacecharge wave, pump wave and scattered wave correspondingly, $L$ - normalized length of scattering region.

Equations (2) possess the following integrals:

$$
A_{i}^{2}-A_{s}^{2}=A_{i 0}^{2}-A_{s 0}^{2} \quad A_{s}^{2}+A_{b}^{2}=A_{s 0}^{2}
$$

where $A_{s 0}=\left.A_{s}\right|_{\xi=0}$ is the signal wave amplitude at the output of the electrodynamic system. Taking into account (3), the equation for the signal wave amplitude reduced to the form:

$$
\frac{d A_{s}}{d z}=-\sqrt{A_{i 0}^{2}-A_{s 0}^{2}+A_{s}^{2}} \sqrt{A_{s 0}^{2}-A_{s}^{2}}
$$

In the steady state oscillations regime, the longitudinal distributions of the wave amplitudes are represented by Jacobi elliptic functions (Fig.2).

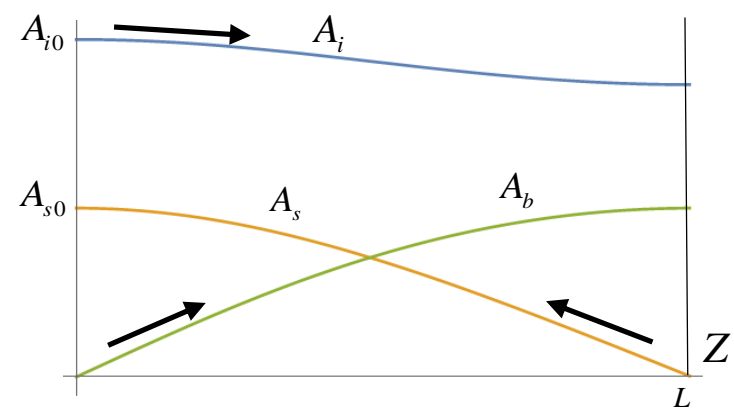

Fig. 2. Longitudinal amplitude distributions in the steadystate oscillations regime

For instance, integrating (4) yields:

$$
A_{s}=A_{s 0} c n\left[A_{i 0} z, \sqrt{\Re}\right]
$$

Here $\mathfrak{R}=A_{s 0}^{2} / A_{i 0}^{2}$ is the quantum yield (the number of pump quants transformed into the signal quants), to be found from equation:

$$
A_{i 0} L=K(\sqrt{\Re})
$$




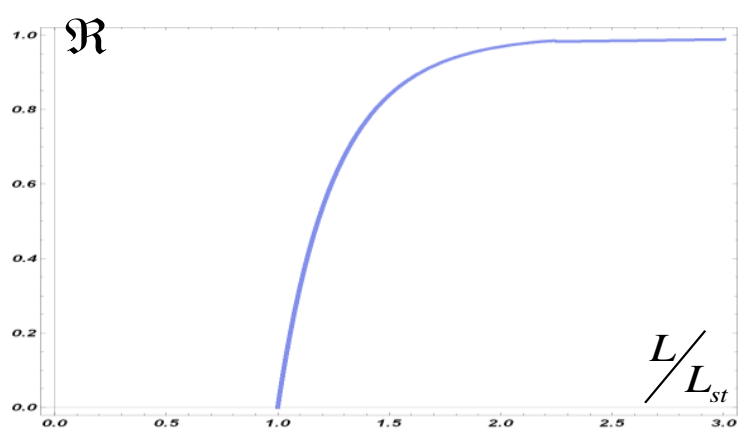

Fig. 3. Dependence of quantum yield on length of scattering region

where $K(x)$ is the complete elliptic integral of the first kind. Fig. 3 shows that quantum yield tends asymptotically to unity as the length of scattering region $L$ increases and when $L$ is two times greater than starting value $L_{s t}$ almost all pump power transferred to the scattered signal. Starting value $L_{s t}$ can be found from the self-excitation condition, derived from (6) at $\mathfrak{R} \rightarrow 0$ :

$$
A_{i 0} L_{s t}=\pi / 2
$$

In physical variables, equation (7) can be rewritten for the oscillator starting current:

$$
I_{s t}=2 \pi^{4} \cdot I_{0} \frac{P_{0}^{2}}{P^{2}} \cdot \frac{\omega_{i}^{2} h_{s}^{2} h_{i}^{2}}{T^{4} \omega_{c}^{4} l^{4}} \cdot \beta_{\| 0}^{7} \cdot \frac{r_{w}^{8} D_{1}\left(N_{s}^{m_{s}, p_{s}}\right)^{2}\left(N_{i}^{m_{i}, p_{i}}\right)^{2}}{r_{b}^{2}}
$$

Here $l$ - the length of scattering region, $T$ - coupling coefficient, $I_{0}=17 \mathrm{kA}, P_{0}=8.7 G W, \quad D_{1}$ - spacecharge forces depression coefficient, $N_{j}^{m_{j}, p_{j}}$ - waveguide mode norm and $P$ is the input pump power.

Estimations were carried out for the input pump power $P=1 \mathrm{MW}$ at wavelength $\lambda_{i}=2 \mathrm{~mm}$. Radius and length of the scattering section were chosen to be $r_{w}=6 \mathrm{~mm}$ and $l=30 \mathrm{~cm}$, correspondingly. Magnetic field $H_{0}$ was taken $3 \%$ off cyclotron resonance value. Both pump and scattered waves were assumed to be $T E_{1,1}$ modes of the cylindrical waveguide. Calculations show (Fig.4) that there exists an optimal radius of the electron beam $r_{b_{\min }}$ providing the minimum value of the starting current. For $T E_{1,1}$ mode $r_{b_{\min }}=1.6 \mathrm{~mm}$.

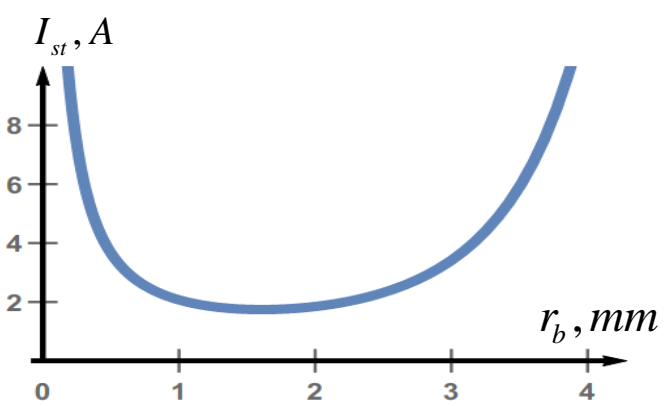

Fig. 4. Dependence of the starting current on the electron beam radius
Further calculations were carried out for $r_{b}=r_{b_{\text {min }}}$. As can be seen from (Fig.5-a), in the case when electron energy is in the range of values $U=2-20 \mathrm{keV}$ starting current does not exceed $6 \mathrm{~A}$ and remains much less than the limiting current of the cylindrical waveguide $I_{\max }$. The magnitude of frequency tuning (Fig.1.4-b), is found from the synchronism condition (1) and is $20-40 \%$ of the pumping frequency, when electron energy is varied in the same interval of electron energies as in (Fig.1.4-a). The change in the frequency of the scattered wave in the range of $5-7 \%$ is achieved by varying the electron energy within the limits of $\pm 2.5 \mathrm{keV}$.
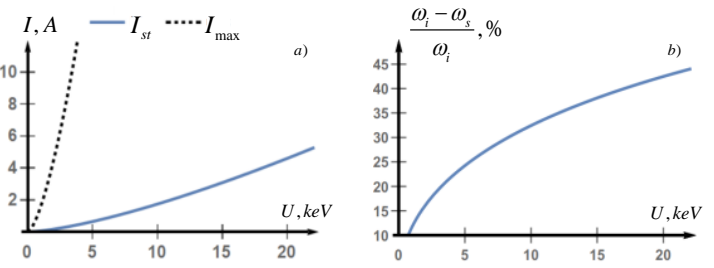

Fig. 5. a) Dependence of limiting (dotted curve) and starting (solid curve) current on electron beam energy, b) Dependence of relative magnitude of frequency tuning on electron beam energy

Magnitude estimations of the electric field on the waveguide surface shows that its values does not exceed $17 \mathrm{kV} / \mathrm{cm}$ and therefore breakdown will not develop. Also an important parameter for estimation is the typical scale of the fields insertion $L_{s w}$. To obtain radiation on the combination synchronism, without oscillations at cyclotron resonance $\omega_{s}-h_{s} V_{\|} \approx \omega_{H}$, an adiabatically smooth insertion of the fields is necessary $\left(\Omega-\omega_{H_{0}}\right) L_{s w} V_{\| 0}^{-1} \sim 2 \pi$, when only stimulated oscillations of the electrons are excited. The estimation of the length $L_{s w}$ for the electron energy range $U=2-20 \mathrm{keV}$ shows that $L_{s w}$ it is within the limits of $2.5-3 \mathrm{~cm}$.

This work was supported by the Russian Scientific Foundation (RSCF), Grant No. 16-42-01078.

\section{References}

1. A.G. Litvak, G.G. Denisov, et al. Development in Russia of $170 \mathrm{GHz} / 1 \mathrm{MW} / \mathrm{CW}$ Gyrotron for ITER // 2011 International Conference on Infrared, Millimeter and Terahertz Waves. 2-7 Oct. 2011. Houston, USA.

2. H. Zohm, M. Thumm. On the use of step-tunable gyrotrons in ITER. // Journal of Physics: Conference Series. Vol. 25. 2005. pp. 274-282.

3. V. M. Malkin, G. Shvets, N.J. Fisch. Fast Compression of Laser Beams to Highly Overcritical Powers. // Physics Review Letters. Vol. 82. No. 22. 1999. pp. 4448-4451.

4. I.Y. Dodin, G.M. Fraiman, V.M. Malkin, N.J. Fisch. Amplification of Short Laser Pulses by Raman Backscattering in Capillary Plasmas. // Journal of Experimental and Theoretical Physics. Vol. 95. No. 4. 2002. pp. 625-638.

5. A.A. Balakin, N.J. Fisch, G.M. Fraiman, V.M. Malkin, Z. Toroker. Numerical modeling of quasitransient backward Raman amplification of laser pulses in moderately undercritical plasmas with multicharged ions. // Physics of Plasmas. Vol. 18. 2011. pp. 102311/1-7. 